\title{
Pourquoi faut-il modifier l'article constitutionnel?
}

\section{Dorothea Wunder}

PD Dr, Centre de Procréation Médicalement Assistée et Endocrinologie Gynécologique (CPMA), Lausanne

Il existent énormément de demi-vérités et de fausses croyances sur ce thème compliqué, ce qui suscite beaucoup de craintes et de grandes peurs. La dignité humaine et le respect de la vie humaine seront tout à fait conservés avec la modification de l'article de la Constitution et le cadre de la nouvelle loi suisse sur la médecine de la reproduction empêchera les dérives.

Je suis gynécologue et depuis 1999 sous-spécialisée dans le domaine de la médecine de reproduction et d'endocrinologie gynécologique. Il peut paraître paradoxal au premier regard, mais quoique je sois pour la modification de l'article constitutionnel, je suis au fond d'accord avec les arguments des opposants: La dignité humaine et le respect de la vie humaine sont des principes fondamentaux, il faut agir contre les tendances d'eugénisme, etc. Comment cette contradiction apparente est-elle possible? La raison en sont à mon avis énormément de demi-vérités et de fausses croyances sur ce thème compliqué, ce qui suscite beaucoup de craintes et de grandes peurs.

\section{Résumé du sujet}

Le peuple suisse vote le 14.6.2015 sur la possibilité future de mettre en culture pour chaque cas individuel autant d'ovules fécondés («imprégnés») que nécessaires pour un traitement optimal.

Actuellement, au maximum 3 ovules fécondés peuvent être mis en culture, les surnuméraires devant être congelés au stade d'ovocyte imprégné. Entre le stade d'ovocyte fécondé et d'embryon à une cellule (dès la

A l'avenir non plus, il n'y aura pas de «stockage illimité» d'embryons.

fusion du noyau féminin et masculin, il s'agit par définition déjà d'un embryon) seulement quelques heures s'écoulent. Le nombre d'ovules imprégnés mis en culture sera aussi limité à l'avenir, ne serait-ce que pour des raisons médicales (risque de syndrome d'hyperstimulation en cas de stimulation ovarienne incontrôlée). De plus, un nombre maximal (12) sera déter- miné par la loi. Autrement dit, il n'y aura pas de cryoconservation excessive d'un nombre illimité d'embryons dans le futur, comme beaucoup d'opposants l'imaginent. Par ailleurs, il faut relever que jusqu'à aujourd'hui, il n'y avait pas non plus de cryoconservation excessive d'un nombre illimité d'ovocytes imprégnés, bien que ce nombre ne soit actuellement pas fixé par la loi, mais pour des raisons médicales et éthiques.

\section{Les avantages}

Le premier grand avantage du changement de la Constitution concerne tous les couples qui font un traitement de fécondation in-vitro (FIV): en congelant des embryons au stade de plusieurs cellules, il sera possible, par le transfert de l'embryon avec les meilleures chances d'implantation, d'arriver aux mêmes chances de grossesse qu'avec un transfert de 2 embryons qui, au stade d'ovocyte fécondé, ont été mis en culture "par hasard» et transférés par la suite. Ainsi, il sera possible de réduire le taux de grossesses multiples après FIV de 15-20\% aujourd'hui, à <5\%, pratique appliquée en Scandinavie avec succès depuis 15 ans. Par la réduction du nombre de grossesses multiples, le risque de prématurité, de complications néonatales et maternelles graves, ainsi que les coûts de la santé peuvent être diminués considérablement.

Le deuxième avantage du changement de la Constitution est qu'il permettra d'effectuer le diagnostic préimplantatoire (= DPI, détection des maladies génétiques graves dans le génome de l'embryon au stade de plusieurs cellules avant l'implantation) aussi en Suisse. Le DPI a été développé il y a environ 20 ans et est effectué dans presque tous les pays européens depuis des décennies. Il faut savoir que le diagnostic prénatal est 


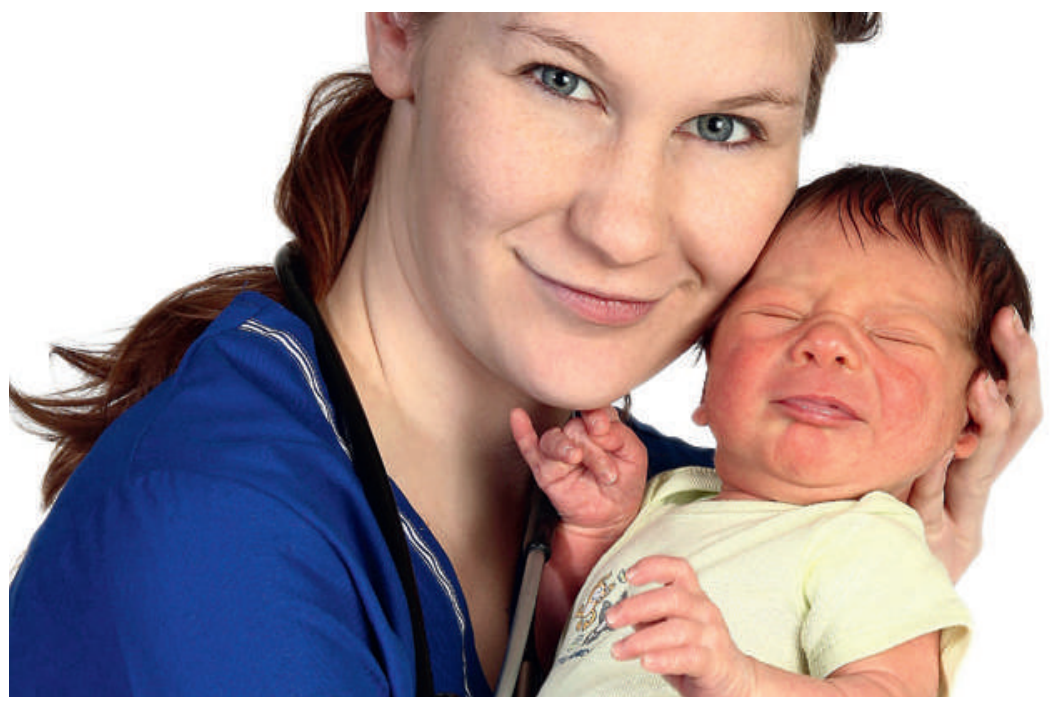

Quel cadeau: un bébé en bonne santé!

autorisé en Suisse et toute grossesse peut être interrompue jusqu'à 3 mois (après la finalisation de l'organogenèse, au stade de développement d'un fœtus), sans justification.

Ainsi, il y aura deux grands avantages grâce à la modification de l'article de la Constitution: Les couples infertiles qui doivent recourir à un traitement de FIV auront de meilleures chances d'avoir un enfant en bonne santé (ceci est prouvé par de multiples études scientifiques) et les couples porteurs d'une maladie héréditaire grave et incurable, qui ont très souvent déjà un enfant handicapé et pour lesquels il est exclu, pour des raisons psychiques et éthiques, d'avoir un deuxième enfant malade, pourront éviter un avortement au-delà de 3 mois de grossesse en suivant le traitement (lourd) d'une fécondation in-vitro avec DPI.

Regardons de plus près les arguments des opposants.

\section{Les arguments des opposants}

- Stockage illimité des embryons qui seront ensuite éliminés au laboratoire: Je serais aussi contre un stockage d'un nombre illimité des embryons. A l'avenir non plus, comme mentionné plus haut, il n'y aura pas de «stockage illimité» d'embryons. De même, les couples auront tous les choix possibles (congélation des embryons ou des ovocytes fécondés, d'ovocytes non-fécondés, voire une stimulation de seulement 2 à 3 ovocytes et ainsi pas de congélation); personne ne sera obligé de congeler des embryons!

- «Un biologiste non impliqué trie au laboratoire les embryons et les jette à son gré; il décide de la valeur ou du peu de valeur de la vie.» Et: "Un acte de mise à mort, qui pourrait à la rigueur encore être évité en cas d'avortement, se passe de manière anonyme en cas de
DPI. Les embryons, pourvus de toutes les qualités pour la vie, se retrouvent dans l'évier du laboratoire»: Quelle vision d'horreur, je serais aussi contre. En réalité, des indications médicales et légales très claires définiront quand un DPI pourra être effectué. En outre, au cours de discussions de plusieurs heures avec les couples avant un DPI, il sera minutieusement défini dans quelles circonstances un embryon ne sera pas transféré dans l'utérus. De plus, il est à mettre en avant qu'il s'agit de couples responsables, avec une expérience de vie douloureuse qui leur permet de juger au mieux leur situation individuelle et de savoir s'ils se sentent capables d'élever (encore) un (autre) enfant gravement malade ou non. Chez les couples qui assument un DPI, il s'agit de parents qui ne pourraient pas faire face à l'arrivée d'un (autre) enfant gravement malade; après un diagnostic prénatal, ils se décideraient, le cœur lourd, pour un avortement. La loi suisse autorise aujourd'hui les couples, suite au diagnostic prénatal avéré d'un handicap grave du fotus, de décider librement de faire ou ne pas faire un avortement (théoriquement jusqu'à la $23^{\mathrm{e}}$ semaine de grossesse), mais personne n'est forcé de faire un diagnostic prénatal. En cas d'autorisation du DPI, tout comme c'est le cas aujourd'hui, les couples pourront continuer à décider librement ce qui est juste pour eux: accepter un (autre) enfant gravement malade, faire le DPI, faire un diagnostic prénatal, un avortement, pas de diagnostic génétique du tout et simplement espérer, renoncer à d'autres enfants, etc.

- L'embryon peut seulement survivre s'il réussit un examen, il s'agit d'une conception à l'essai: Oui, la "conception à l'essai» est une réalité depuis l'introduction des techniques de diagnostic prénatal et la possibilité de l'avortement, ceci indépendamment du DPI. La décision de faire ces tests ou de ne pas les faire, d'avorter ou de ne pas avorter, appartient aux futurs parents. Cette réalité doit être acceptée et si dans ce

\section{Il s'agit de couples responsables qui peuvent} juger au mieux leur situation et savent s'ils se sentent capables d'élever (encore) un (autre) enfant gravement malade ou non.

contexte il est clair qu'un couple individuel ne peut pas accepter un enfant avec une maladie génétique grave, il faut se poser la question suivante: est-ce qu'il est plus grave d'avorter un fotus après le développement des organes ou d'éviter l'implantation de l'embryon porteur de la maladie génétique grave au stade de quelques cellules (et d'éviter ainsi un avortement)? 
- Les enfants handicapés (et leurs parents) seront de plus en plus stigmatisés: C'est une fausse croyance. L'acceptation des handicapés dans notre société n'a pas diminué depuis l'introduction du diagnostic prénatal, elle a au contraire augmenté. En outre, l'acceptation de personnes handicapées ne dépend pas de leur nombre. Rien n'indique que cela changera en Suisse après l'autorisation du DPI, plus particulièrement si l'on considère le très petit nombre de parents porteurs d'une maladie génétique grave et le faible pourcentage d'enfants FIV en général (même en cas de screening des aneuploïdies - ce qui aujourd'hui est ni effectué de routine, ni «State of the Art» à l'étranger - on diagnostiquerait par an en Suisse au maximum un embryon avec une trisomie 21).

- Le bonheur ou la souffrance ne se diagnostiquent pas: C'est absolument juste. En plus, un diagnostic génétique n'exclue ni tous les handicapés ni toutes les malformations, sans parler des possibles complications de la grossesse telles que prématurité, morbidité néonatale, etc.! Il n'y a pas de certitude dans la vie, avec ou sans diagnostic génétique, et il y aura toujours des personnes handicapées, avec des maladies génétiques graves, avec des malformations, des maladies physiques et psychiques sévères, etc. Mais est-ce que cette réalité peut être utilisée en tant qu'argument pour priver de la possibilité du DPI les couples porteurs d'une maladie génétique grave, souhaitant éviter à leur enfant et leur famille de souffrir?

- Le screening préimplantatoire est un screening génétique total et sera réalisé sur tous les embryons: Non, quelle horreur de s'imaginer que chaque embryon devra à l'avenir être soumis à un examen génétique complet et selon le résultat être éliminé. Il n'en est rien, en Europe aussi. II s'agit d'une technique très délicate et non pas d'un "State of the Art». Par ailleurs, un screening préimplantatoire n'est pas un "screening génétique complet»; on recherche les mêmes anomalies chromosomiques que lors d'un diagnostic prénatal. Un screening d'aneuploïdie serait indiqué seulement dans des cas particuliers, par exemple chez une patiente infertile devant suivre un traitement de FIV pour concrétiser son désir d'enfant, présentant un risque élevé de trisomie 21, mais qui ferait une interruption de grossesse en cas de trisomie 21 avérée. En évitant le transfert d'un embryon avec trisomie 21, on pourrait lui épargner une interruption de grossesse.

- Le screening préimplantatoire est dangereux et représente un risque d'eugénisme: On ne pourra jamais écarter à 100\% le risque d'abus futurs dans le monde. Mais est-ce que l'hypothèse d'un abus quelque part dans le monde peut être un argument pour interdire cette technique, qui pour certains couples représente une bénédiction, qui, en outre, sera réglementée par notre loi suisse sur la procréation médicalement assistée grâce à des indications soigneusement définies, sévères et éthiquement acceptées? L'histoire mondiale a montré que le risque de pensée eugénique existe depuis longtemps. Il trouve son origine non pas dans les (nouvelles) techniques génétiques, mais dans des pensées idéologiquement erronées et se rencontre avant tout dans des régimes totalitaires. Il est du devoir de l'Etat de stopper d'éventuelles tendances eugéniques. Ces craintes hypothétiques ne doivent cependant pas être utilisées comme argument pour interdire aux couples la possibilité de bénéficier de ce traitement médical. Surtout si l'on considère que le traitement FIV signifie pour la femme concernée une thérapie longue, physiquement et psychiquement stressante, invasive et lourde dont le succès $(=$ la naissance d'un enfant en bonne santé) est tout autre que garanti. A part cela, les mêmes «risques d'eugénisme» se posent théoriquement en Suisse pour le diagnostic prénatal qui peut être effectué après conception spontanée, sans traitement FIV, pénible, lourd et coûteux.

\section{Résumé}

En résumé, on peut dire que la dignité humaine et le respect de la vie humaine seront tout à fait conservés avec la modification de l'article de la Constitution et que le cadre de la nouvelle loi suisse sur la médecine de la reproduction empêchera les dérives. Grâce à la modification de l'article de la Constitution, les grossesses multiples après FIV pourront être réduites d'aujourd'hui $20 \%$ à moins de $5 \%$. En réduisant les grossesses multiples, on diminue considérablement le risque de graves complications néonatales et maternelles ainsi que les coûts de la santé. En plus, le DPI pourra éviter des avortements chez les couples porteurs d'une maladie génétique grave. 